\title{
Adaptive optics imaging of the MBM 12 association
}

\section{Seven binaries and an edge-on disk in a quadruple system ${ }^{\star}$}

\author{
G. Chauvin ${ }^{1}$, F. Ménard ${ }^{1}$, T. Fusco ${ }^{2}$, A.-M. Lagrange ${ }^{1}$, J.-L. Beuzit ${ }^{1}$, D. Mouillet ${ }^{3}$, and J.-C. Augereau ${ }^{4}$ \\ ${ }^{1}$ Laboratoire d'Astrophysique, Observatoire de Grenoble, 414, rue de la piscine, BP 53, 38041 Grenoble Cedex 9, France \\ 2 Office National d'Études et de Recherches Aérospatiales, Département d'optique théorique et appliquée, BP 72 , \\ 92322 Châtillon Cedex, France \\ 3 Laboratoire d'Astrophysique, Observatoire Midi-Pyrénées, BP 826, 65008 Tarbes, France \\ ${ }^{4}$ DSM/DAPNIA/Service d'Astrophysique, CEA/Saclay, 91191 Gif-sur-Yvette, France
}

Received 23 April 2002 / Accepted 29 July 2002

\begin{abstract}
We report adaptive optics (AO) observations of the young and nearby association MBM 12 obtained with the CanadaFrance-Hawaii Telescope. Our main observational result is the discovery of six new binary systems, LkH $\alpha 264, \mathrm{E} 0255+2018$, RX J0255.4+2005, S18, MBM 12-10, RX J0255.3+1915, and the confirmation of HD 17332, already known as a binary. We also detected a possible quadruple system. It is composed of the close binary $\mathrm{LkH} \alpha 263 \mathrm{AB}$ (separation of $\sim 0.41^{\prime \prime}$ ), of $\mathrm{LkH} \alpha 262$ located $\sim 15.25^{\prime \prime}$ from $\mathrm{LkH} \alpha 263 \mathrm{~A}$, and of $\mathrm{LkH} \alpha 263 \mathrm{C}$, located $\sim 4$. $1^{\prime \prime}$ from $\mathrm{LkH} \alpha 263 \mathrm{~A}$. A preliminary study of the binary fraction suggests a binary excess in the MBM 12 association as compared to the field and IC 348. Because of the high binarity rate, previous estimations of spectral types and measurements of IR excesses for several candidate members of MBM 12 have to be revised. $\mathrm{LkH} \alpha 263 \mathrm{C}$ is a nebulous object that we interpret as a disk oriented almost perfectly edge-on and seen in scattered light. This object has already been reported by Jayawardhana et al. (2002). Scattered light models allow us to estimate some of the structural parameters (i.e. inclination, diameter and to a lesser extent dust mass) of the circumstellar disk. We find an inclination of $89^{\circ}$ and a outer radius for the disk, $\sim 165 \mathrm{AU}$ if the distance to MBM 12 is $275 \mathrm{pc}$. With the present data set, we do not attempt to re-assess the distance to MBM 12. We estimate however that the distance to the candidate member RX J0255.3+1915 is $d>175$ pc.
\end{abstract}

Key words. stars: pre-main sequence - stars: binaries: general - stars: planetary systems: protoplanetary disks stars: circumstellar matter - stars: low-mass, brown dwarfs

\section{Introduction}

Young and nearby open clusters are ideal laboratories to study the formation and evolution of solar-like stars. Due to their proximity, circumstellar disks are examined with finer resolution and lower mass objects can be detected. In the best case, one can also hope to find giant planets orbiting cluster members, using current instruments. It is therefore no surprise that vast efforts were made to discover new nearby associations in recent years. Today, seven nearby associations, open clusters, or so-called "groups" are known that are closer than $100 \mathrm{pc}$ from the Sun. They are the TW Hydrae and $\eta$ Chamaeleontis associations (Kastner et al. 1997; Mamajek et al. 1999, respectively), Horologium A and Tucana associations (Torres et al. 2000; Zuckerman et al. 2000, respectively).

Send offprint requests to: G. Chauvin, e-mail: gchauvin@obs.ujf-grenoble.fr

* Based on data collected at the Canada-France-Hawaii Telescope. The CFHT corporation is funded by the Governments of Canada and France, and by the University of Hawaii.
Zuckerman et al. (2001a) recently proposed that they are part of the same stream. The Capricornus association has been described by van den Ancker et al. (2000). A loose group was also identified by Zuckerman et al. (2001b) around the nearby and well-known star $\beta$ Pictoris.

The seventh nearby association is found in the high latitude molecular cloud complex made of clouds number 11, 12 and 13 in the list of Magnani et al. (1985). This complex was identified earlier by Lynds (1962) and is comprised of the dark nebulae L1453, L1454, L1457 and L1458. Clumpy $100 \mu \mathrm{m}$ infrared emission is detected by IRAS at the position of the dark nebulae. The molecular clouds also cast a deep shadow on the X-ray background detected by ROSAT (Snowden et al. 1993). The total mass of the entire complex is estimated to be 30$200 M_{\odot}$ based on ${ }^{12} \mathrm{CO},{ }^{13} \mathrm{CO}$ and $\mathrm{C}^{18} \mathrm{O}$ maps (Pound et al. 1990; Zimmermann \& Ungerechts 1990). This association of young stars, which we call MBM 12 from now on, is particularly interesting because it is more compact than the others, with all the stars located within the boundaries of the small molecular cloud complex. Although it is not clear whether the 
cloud is bound by gravity or not, it was rapidly shown to contain many IRAS sources and optically revealed T Tauri stars (Magnani et al. 1990) proving its capacity to form solar-like stars. The list of members of MBM 12 now extends to 14 (Hearty et al. 2000a; Luhman 2001). MBM 12 is also interesting because, among the seven young nearby associations known, it appears to be the youngest with an estimated age of $2_{-1}^{+3}$ Myr (Luhman 2001).

First estimations of its distance placed it at $65 \mathrm{pc}$ from the Sun (Hobbs et al. 1986; Hobbs et al. 1988), a result later confirmed by Hearty et al. (2000b) with Hipparcos trigonometric parallaxes of fore- and background stars. Luhman (2001) revised this distance, suggesting 275 pc based on 2MASS photometry and optical spectroscopy. Although the exact distance to the association is still being debated, at the distance of $65 \mathrm{pc}$, MBM 12 would be the nearest star forming molecular cloud known to date.

In MBM 12, the first investigation for protoplanetary disks was done by Jayawardhana et al. (2001). Significant midIR excess of six candidate members ( $\mathrm{LkH} \alpha 263, \mathrm{LkH} \alpha 262$, $\mathrm{LkH} \alpha$ 264, E 0255+2018, RX J0258.3+1947 and S18) are reported and attributed to the presence of circumstellar disks. To test this interpretation and detect the possible existence of circumstellar matter around these stars, high resolution imaging is needed. In this paper we present the results of near-infrared AO observations carried out at the $3.6 \mathrm{~m}$ Canada-France-Hawaii Telescope. Four mid-IR excess members are resolved as binary systems and a protoplanetary disk is detected around the binary system $\mathrm{LkH} \alpha 263 \mathrm{AB}$. We report also $\mathrm{AO}$ observations of four other candidate members (RX J0255+2005, MBM 1210, RX J0255.3+1915 and HD 17332) which are resolved as binary systems. All companion candidates presently detected need to be confirmed or rejected later by proper motion or spectroscopy. The observations are described in the next section. Section 3 presents the results. The binary fraction studied for observed MBM 12 members, the implication of the high rate we found, the distance and a scattered light model for the edgeon disk $\mathrm{LkH} \alpha 263 \mathrm{C}$ are respectively discussed in Sects. 4-7.

\section{Adaptive optics imaging}

The AO observations were carried out on 2001 November 29th and 30th and on December 2nd at the Canada-France-Hawaii telescope. KIR, the facility $1024 \times 1024$ near-infrared camera dedicated to AO, was used. The pixel scale on the detector is $0.0348^{\prime \prime} / \mathrm{px}$, giving a total field of view of $35.6^{\prime \prime} \times 35.6^{\prime \prime}$. The observations were obtained in the $J, H$ and $K$ s standard nearinfrared bands for eight fields centered on the eight brightest members of MBM 12 and consisted of nine previously known targets. The field centers are listed in Table 1. The ninth target is $\mathrm{LkH} \alpha 262$, in the field of $\mathrm{LkH} \alpha 263$.

$\mathrm{LkH} \alpha 264$ was observed with the coronographic mode of $\mathrm{GRIF}^{1}$ to improve the dynamic range, hence better the detectability of the faint circumstellar environments. The diameter of the occulting mask was $0.9^{\prime \prime}$. The photometric

\footnotetext{
1 http: //www. cfht . hawaii. edu/Instruments/ Spectroscopy/GriF/
}

standards FS 121 and FS 129 from the UKIRT faint standard list were observed as photometric and PSF standards. They complement, at low level in the seeing halo, the PSF estimation reconstructed analytically from the wavefront sensor residual errors (Véran 1997). The usual sky background subtraction, cosmic ray hits correction, and removal of detector signatures (i.e., flat field, bias, deviant pixels) were performed. The deconvolution algorithms of Véran et al. (1998) and the myopic deconvolution algorithm MISTRAL (Conan et al. 2000) were then used to obtain the flux ratios and the separations of the binaries and high angular resolution images of the protoplanetary disk $\mathrm{LkH} \alpha 263 \mathrm{C}$ in JHKs.

\section{Results}

\subsection{New binary systems in MBM 12}

We resolved six new binary systems out of the nine MBM 12 members covered by our observations. They are LkH $\alpha$ 264, E 0255+2018, RX J0255.4+2005, S18, MBM 1210, RX J0255.3+1915. We also confirm that HD 17332 is a 3.6" binary. Figures 1 and 2, left panel, show $K$ s-band images of these binaries. E $0255+2018$, RX J0255.4+2005, S18, MBM 12-10 and HD 17332 were deconvolved using the algorithm written by Véran et al. (1998). In the case of RX J0255.3+1915, flux ratio and separation were obtained with the myopic deconvolution algorithm MISTRAL. The companion of $\mathrm{LkH} \alpha 264$ was detected by coronography only. The contrast was estimated a posteriori by aperture photometry on normal images obtained without a coronographic mask using an aperture of $R_{\text {ap }}=0.7^{\prime \prime}$ (see Fig. 2, left panel). We present our photometric results in Table 1. The first three columns give the name and the coordinates, in the equatorial system, of the photocenter of the unresolved system given by Luhman (2001). Columns 4-6 give the relative brightness of the companion candidate with respect to the primary in $J, H$, and $K$ s respectively. The last two columns give the position angle and separation at which the companion was detected.
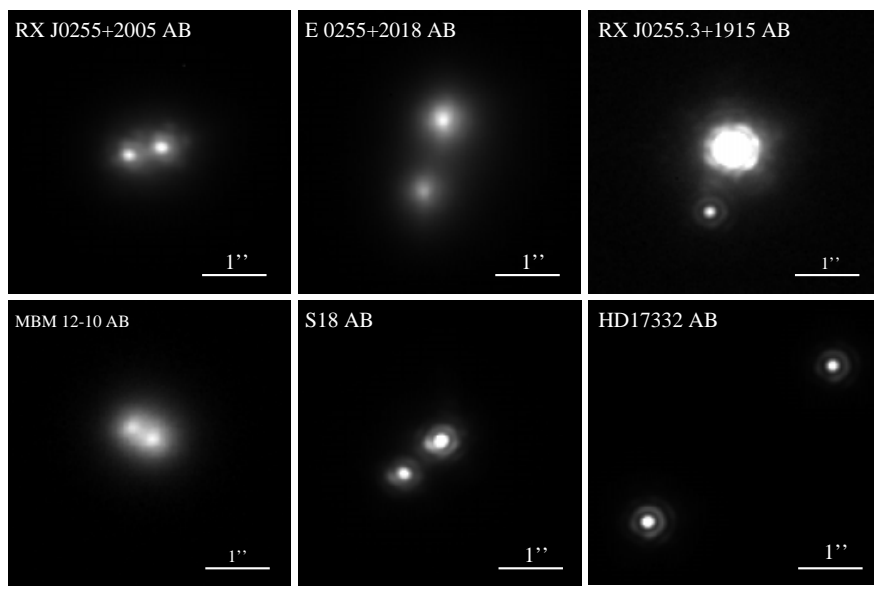

Fig. 1. AO observations in $K$ s-band of the six close binaries: $\mathrm{E} \quad 0255+2018, \mathrm{RX} \mathrm{J} 0255.4+2005, \mathrm{~S} 18$, MBM 12-10, RX J0255.3+1915 and HD 17332. North is up and east is left. Each image is presented in linear stretch. 
Table 1. Contrasts and separations of the binary systems observed in the MBM 12 Association.

\begin{tabular}{llllllll}
\hline \hline Name & $\alpha(2000)$ & $\delta(2000)$ & $\begin{array}{l}\Delta J \\
(\mathrm{mag})\end{array}$ & $\begin{array}{l}\Delta H \\
(\mathrm{mag})\end{array}$ & $\begin{array}{l}\Delta K \mathrm{~s} \\
(\mathrm{mag})\end{array}$ & $\begin{array}{l}\text { PA } \\
\left({ }^{\circ}\right)\end{array}$ & $\begin{array}{l}\text { separations } \\
\left({ }^{\prime \prime}\right)\end{array}$ \\
\hline RX J0255+2005 AB & 025525.78 & 200451.7 & & & $0.13 \pm 0.04$ & $104 \pm 1$ & $0.503 \pm 0.002$ \\
LkH $\alpha$ 263 AB & 025607.99 & 200324.3 & $-0.06 \pm 0.01$ & $-0.21 \pm 0.01$ & $0.18 \pm 0.01$ & $54 \pm 1$ & $0.410 \pm 0.002$ \\
LkH $\alpha$ 264 AB & 025637.5 & 200538 & & & $6.75 \pm 0.2$ & $223 \pm 1$ & $9.160 \pm 0.034$ \\
E 0255+2018 AB & 025811.2 & 203004 & & & $0.50 \pm 0.02$ & $165 \pm 1$ & $1.108 \pm 0.009$ \\
MBM 12-10 AB & 025821.10 & 203252.7 & & & $0.03 \pm 0.03$ & $61 \pm 1$ & $0.390 \pm 0.007$ \\
S18 AB & 030221.1 & 171035 & $0.56 \pm 0.02$ & $0.42 \pm 0.02$ & $0.69 \pm 0.01$ & $130 \pm 1$ & $0.753 \pm 0.001$ \\
RX J0255.3+1915 AB (b) & 025516.5 & 191502 & $3.61 \pm 0.05$ & $4.2 \pm 0.03$ & $3.64 \pm 0.03$ & $161 \pm 1$ & $1.014 \pm 0.002$ \\
HD17332 AB (a) & 024727.3 & 192224 & & & $0.42 \pm 0.01$ & $311 \pm 1$ & $3.63 \pm 0.006$ \\
\hline
\end{tabular}

a Narrow Band Filter $\mathrm{H}_{2}$ used.

b Deconvolved by MISTRAL.
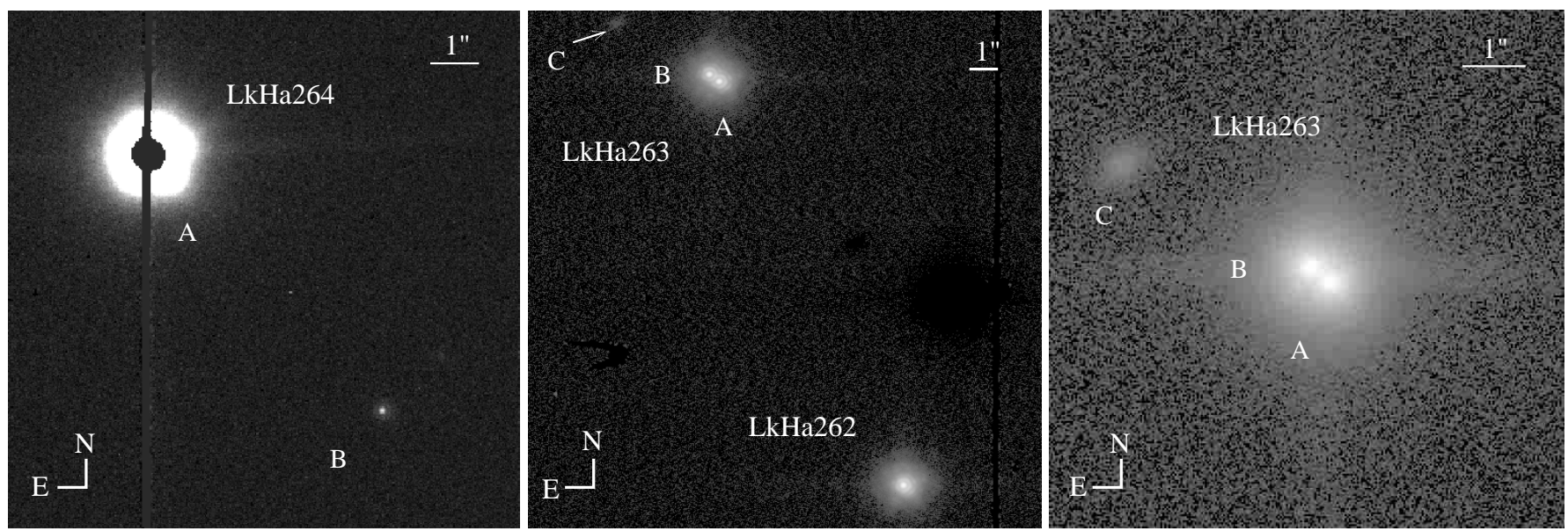

Fig. 2. Left: coronographic AO observation of $\mathrm{LkH} \alpha 264 \mathrm{~A}$ in $K$ s-band. A faint companion candidate LkH $\alpha 264 \mathrm{~B}$ appears at $9.16^{\prime \prime}$ with a position angle of $223^{\circ} \pm 1$. Middle: $\mathrm{AO}$ observation in $H$-band of the quadruple system composed of $\mathrm{LkH} \alpha 262, \mathrm{LkH} \alpha 263 \mathrm{AB}$ and $\mathrm{LkH} \alpha 263 \mathrm{C}$. Right: zoom of the close binary $\mathrm{LkH} \alpha 263 \mathrm{AB}$ and the young protoplanetary disk $\mathrm{LkH} \alpha 263 \mathrm{C}$. Images are presented in linear stretch.

\section{2. $L k H \alpha$ 263-262, a quadruple system?}

In the field centered on $\mathrm{LkH} \alpha 263$, we detected four stars that may form a physical system.

\subsection{1. $\mathrm{LkH} \alpha 263 \mathrm{AB}$ and $\mathrm{LkH} \alpha 262$}

Similarly to Jayawardhana et al. (2002) we resolve LkH $\alpha 263$ into a close binary, with a projected separation of $0.41^{\prime \prime}$ and position angle of $54^{\circ} \pm 1$, measured East of North. Further out to the North-East, another fainter and very nebulous object appears. We call it $\mathrm{LkH} \alpha 263 \mathrm{C}$. It lies at $4.1^{\prime \prime} \pm 0.05$ and at position angle $61^{\circ} \pm 1$ from $\mathrm{LkH} \alpha 263 \mathrm{~A}$. Still further out, and possibly not bound to the system, $\mathrm{LkH} \alpha 262$ lies at $15.25^{\prime \prime} \pm 0.05$ and position angle $205^{\circ} \pm 1$ degrees from $\mathrm{LkH} \alpha 263 \mathrm{~A}$.

Figure 2 shows two $H$-band images of this system. The right panel is a zoom on the close binary $\mathrm{LkH} \alpha 263 \mathrm{AB}$ and the nebulous $\mathrm{LkH} \alpha 263 \mathrm{C}$. The middle panel presents the whole system, with $\mathrm{LkH} \alpha 262$ at the bottom right and $\mathrm{LkH} \alpha 263 \mathrm{C}$ on the edge of the frame, at top left.

Flux ratio and separation of $\mathrm{LkH} \alpha 263 \mathrm{AB}$ were obtained using the deconvolution algorithm of Véran et al. (1998) previously discussed. The relative photometry of the pair $\mathrm{LkH} \alpha 263 \mathrm{AB}$ is also reported in Table 1. $\mathrm{LkH} \alpha 263 \mathrm{~B}$ is brighter than $\mathrm{LkH} \alpha 263 \mathrm{~A}$ in $J$ and $H$, but not in $K \mathrm{~s}$.

\subsubsection{The nebulous object $\mathrm{LkH} \alpha 263 \mathrm{C}$}

The appearance of $\mathrm{LkH} \alpha 263 \mathrm{C}$ is reminiscent of that of other young stars with a circumstellar disk seen close to edge-on. It appears very similar to HK Tau/c (Stapelfeldt et al. 1998), HV Tau C (Monin \& Bouvier 2000; Stapelfeldt et al. 2002) and IRAS 04158+2805 (Ménard et al. 2002).

The angular dimensions of the nebulosity associated with $\mathrm{LkH} \alpha 263 \mathrm{C}$ are presented in Table 2. The discovery, and first interpretation of $\mathrm{LkH} \alpha 263 \mathrm{C}$ as an edge-on disk was made by Jayawardhana et al. (2002) from $H$-band AO images. Our diffraction-limited JHKs images are presented in Fig. 3, top panel. They are compatible with the images made by Jayawardhana et al. (2002). The angular resolution of our images is lower however, $0.15^{\prime \prime}$ on the raw frames. Nevertheless, the dark lane typical of optically thick edge-on disks, is present. The advanced deconvolution algorithm MISTRAL was applied to the image to try and recover the maximum spatial 


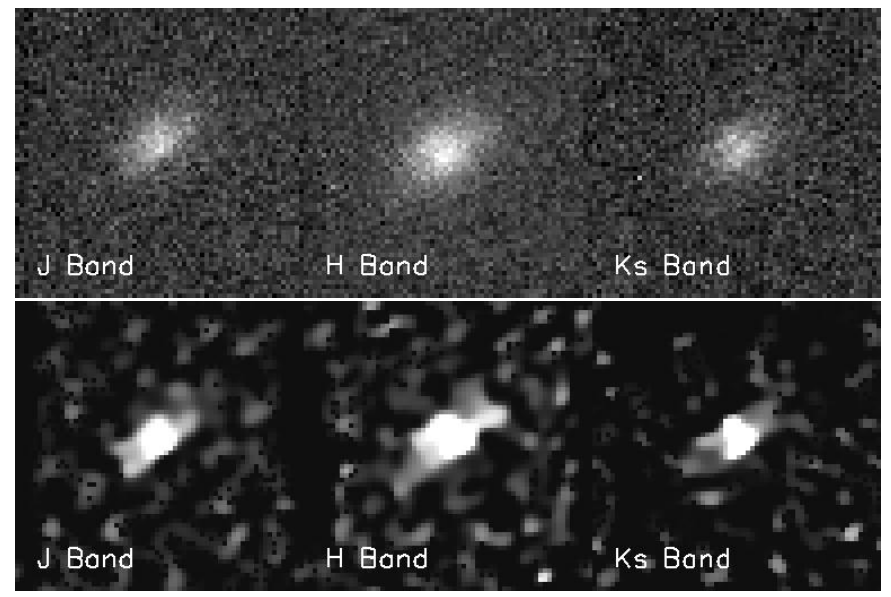

Fig. 3. Top row: raw images of the circumstellar disk surrounding $\mathrm{LkH} \alpha 263 \mathrm{C}$ in $J, H$ and $K$ s (from left to right). Bottom row: deconvolved images. MISTRAL, a myopic deconvolution algorithm was used to produce these $J, H$ and $K$ s images (also from left to right). On all images the field-of-view is $2.2^{\prime \prime}$, with North up and East to the left.

information possible. The signal-to-noise in the images is low and the efficiency limit of MISTRAL is reached. The results are presented in Fig. 3, bottom panel. The parameters given in Table 2 are measured on the deconvolved images.

Table 2. $\mathrm{LkH} \alpha 263 \mathrm{C}$ : parameters of the reflection nebula.

\begin{tabular}{llll}
\hline \hline Filter & $\begin{array}{l}\text { Diameter } \\
\text { arcsec }\end{array}$ & $\begin{array}{l}\text { Brightness } \\
\text { ratio }\end{array}$ & $\begin{array}{l}\text { Separation } \\
\text { arcsec }\end{array}$ \\
\hline$J$ & $0.69 \pm 0.07$ & $1.56 \pm 0.1$ & 0.126 \\
$H$ & $0.87 \pm 0.07$ & $1.32 \pm 0.1$ & 0.148 \\
$K$ s & $0.72 \pm 0.07$ & $1.13 \pm 0.1$ & 0.156 \\
Gemini $H$ & $0.90 \pm 0.06$ & 1.2 & 0.141 \\
\hline
\end{tabular}

The general appearance of all the images is the same, two elongated reflection nebulae, parallel to each other and separated, more or less depending on angular resolution, by a dark lane. The dark lane is conspicuous on the Gemini images of Jayawardhana et al. (2002) but is at the resolution limit on our images, although still detectable.

In all three images the dark lane runs at position angle $128^{\circ} \pm 1$ with the brighter nebula located to the South-West. The diameter of the disk can be measured in all three images as the maximum distance over which the reflection nebula can be detected. The values are quoted in the second column of Table 2. The diameters differ slightly from filter to filter. This variation is mostly due to variations in the signal-to-noise ratio and resolution in the various frames. Our best data set was obtained at $H$-band and, as expected, we find the maximum diameter, at about $0.9^{\prime \prime}$, or $R_{\text {out }}=0.45^{\prime \prime}$ (i.e., an observed outer radius of $124 \mathrm{AU}$, assuming a distance of $275 \mathrm{pc}$, or $30 \mathrm{AU}$ for $65 \mathrm{pc}$ ). This value should be regarded as a lower limit to the disk size since deeper images may reveal the disk farther out. This value is similar to the one extracted from images of Jayawardhana et al. (2002).
In Table 2, the brightness ratio given in the third column is the ratio of the peak pixel value in the South-West brighter nebula to the peak pixel value in the North-East fainter nebula. Those are the two maxima measured along the minor axis of the disk. The separation given in the fourth column is the distance, in arcsec, measured between these two peaks. Typical error bars on the angular measurements are two pixels (i.e., 70 mas). All measurements are made on the deconvolved images.

\section{Binary star fraction in observed MBM 12 members}

From the 14 members of the MBM 12 association discovered by Hearty et al. (2000a) and Luhman (2001), we observed 9 during our AO imaging campaign. We were sensitive to separations ranging from $0.13^{\prime \prime}$ (diffraction limit in $K \mathrm{~s}$ ) to $18^{\prime \prime}$ (half field of view). As we previously mentioned, a quadruple system composed of the two members $\mathrm{LkH} \alpha$ 262-263 and six binaries were detected in classical imaging. In addition, coronographic imaging allowed us to detect another companion candidate around $\mathrm{LkH} \alpha 264$.

To conduct our analysis on the binary star fraction $(b f)$ for the small sample of MBM 12 members observed, we consider only AO classical imaging observations. The averaged detection limit achieved in $K$ s-band as a function of the distance to the star is presented in Fig. 4 with the different companion candidates overplotted (except $\mathrm{LkH} \alpha 262$ as discussed next). To compare the $b f$ of our sample with the one obtained by Duchêne et al. (1999) for the young cluster IC 348 (located at $320 \mathrm{pc}$ and aged of $2 \mathrm{Myr}$ ), we limit our study to the same separation range 32-2560 AU. This means an angular separation range of $0.13^{\prime \prime}-9.3^{\prime \prime}$ if we assume the distance of $275 \mathrm{pc}$ to MBM 12. We also limit the dynamic range to detect binaries with mass ratio $q=0.1$ as Duchêne et al. (1999) did to compare their results to that of the low-mass main sequence (MS) stars, G- and M-dwarfs (Duquennoy \& Mayor 1991; Fischer \& Marcy 1992). Based on mass-luminosity relation at $2 \mathrm{Myr}$ from Baraffe et al. (1998), this mass ratio is equivalent to a $\Delta K \mathrm{~s}=2.9$. Duchêne et al. (1999) conducted their near-IR survey with the same instrument, PUEO/KIR, and the same detection performance was achieved ( 6.5 mag for separation $\left.>1^{\prime \prime}\right)$. In our sample, the 6 close binaries have separations between $0.39^{\prime \prime}$ and $3.63^{\prime \prime}$ and contrast in $\Delta K$ s between 0.03 and 3.64. The three components $\mathrm{LkH} \alpha 263 \mathrm{~B}, \mathrm{LkH} \alpha 263$ $\mathrm{C}$ and $\mathrm{LkH} \alpha 262$ of the visual quadruple system have respectively separation of $0.41^{\prime \prime}, 4.1^{\prime \prime}$ and $15.25^{\prime \prime}$ and contrast of $0.18,5.6$ and -0.52 from $\mathrm{LkH} \alpha 263$ A. According to the separation range we fixed, we consider this system composed of a triple system $\mathrm{LkH} \alpha 263 \mathrm{ABC}$ and a single star $\mathrm{LkH} \alpha 262$. The source $\mathrm{LkH} \alpha 264$ with a faint companion detected in coronagraphy is also considered as a single star.

For the young cluster IC 348, Duchêne et al. (1999) evaluate the $b f\left(=\frac{B+T+Q}{S+B+T+Q} ; B, T\right.$ and $Q$ respectively for Binary, Triple and Quadruple system) at $19 \pm 5 \%$. This is similar to the values of G- and M- dwarfs in the solar neighbourhood (23\% and $18 \%$, respectively) and shows that there is no binary excess in IC 348. Based on the same separation range for a distance 


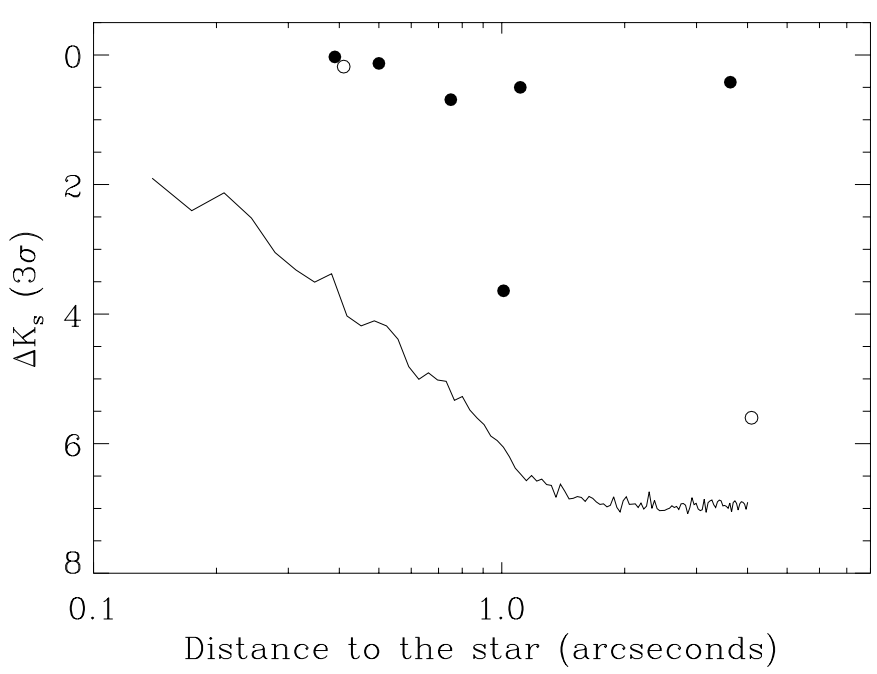

Fig. 4. Averaged detection limit obtained in our classical imaging observations in $K$ s-band. Filled circles are detected binaries around MBM 12 members. Open circles are companions LkH $\alpha 263$ B and $\mathrm{LkH} \alpha 263 \mathrm{C}$ detected around $\mathrm{LkH} \alpha 263 \mathrm{~A}$. $\mathrm{LkH} \alpha 262$, located at $15.25^{\prime \prime}$ from $\mathrm{LkH} \alpha 263 \mathrm{~A}$ is considered as a single star.

of 275 pc to MBM 12 and the same dynamic range, we estimate the $b f$ of our sample to $66 \%$. This suggests a large binary excess. For a smaller distance of $65 \mathrm{pc}$, the separation range becomes 8-604 AU and we still find an excess compared to values of G- and M- dwarfs for the same separation range (28\% and $22 \%$, respectively).

Therefore, this preliminary result suggests a large binary excess in the MBM 12 association and points out the trend presented by Duchêne (1999) and Duchêne et al. (1999) that the binary fraction is inversely correlated with the stellar density. Dense clusters have a binary fraction similar to the field dwarfs, whereas loose young open clusters exhibit binary excesses. Gravitational encounters in dense clusters or specific initial conditions in the parent molecular clouds may lead to these different binary fractions. To confirm this trend for a binary excess in the MBM 12 association, further AO imaging observations of other candidate members are needed.

\section{Impact of the high binarity rate}

\subsection{Relevance of previous spectral type estimations}

Our discovery of frequent visual pairs in MBM 12 may call for a revision of the estimated spectral types in cases where the binarity was unknown before and the contrast between companions is not large enough for one star to dominate in the spectrum used for classification.

The contribution of the companion candidates in the optical spectra of $\mathrm{LkH} \alpha 264$, and RX J0255.3+1915 is small and the spectral types given by Luhman (2001) should stand. Also, we did not detect a companion candidate to $\mathrm{LkH} \alpha 262$, and the spectral types given before also stand.

For all other cases, i.e., LkH $\alpha$ 263, S18, E 0255+2018, RX J0255+2005, and MBM 12-10, the contrast between the primary and the secondary is low enough, less than 0.7 mag (i.e., less than a factor 2 in counts) that the estimated spectral types may be affected if the two stars have different temperatures. This is likely the case for $\mathrm{LkH} \alpha 263$. It is not as clear for the others. A careful re-interpretation of individual spectral types seems in order for this group of objects and the current estimates should be considered as indicative only.

\subsection{The origin of the near-infrared excess}

The presence of an optically undetected companion may also affect measurements of the infrared excess, possibly invalidating the attribution of its origin to the circumstellar medium, i.e., a disk. For MBM 12, Jayawardhana et al. (2001) presented $L$ and $N$-band infrared photometry of 10 stars, including 8 from the sample we observed.

In 6 cases these authors measured large $(K-L)$ and $(K-N)$ color indices, and attributed the origin of the excess to warm dust located in a protostellar disk following, e.g., Kenyon \& Hartmann (1990), and Skrutskie et al. (1990). Of those six sources we observed five, and detected four binaries. Those were unknown when Jayawardhana et al. (2001) performed their analysis and they were all treated as single objects.

If those objects have significantly different spectral types, or temperature, then the amplitude of the color excesses previously attributed the circumstellar environment of a single object may not be valid anymore. The case of $\mathrm{LkH} \alpha 263 \mathrm{AB}$ is interesting. Clearly, the fainter component at $J$ and $H$ becomes the brighter one at $K$ s. It is difficult to extrapolate to longer wavelengths, but most of the color excess may well come from the companion, without the need for a massive protoplanetary disk at all. The other cases are not as clear and a color excess may still be present, despite the presence of a companion. Obviously, new spectroscopic observations combined with high angular resolution are needed to determine the colors and the stellar parameters of each component in the binaries.

\section{The distance to RX J0255.3+1915}

It is beyond the scope of this paper to measure the distance to MBM 12. The molecular cloud appears clumpy and adjacent lines-of-sight projected on the cloud can have very different extinction values, e.g., Bhatt et al. (1994), making it difficult to estimate the distance reliably. In a recent paper, Luhman (2001) revised the distance to the assocation to $275 \mathrm{pc}$. This is significantly farther then the value of $65 \mathrm{pc}$ estimated before by Hobbs et al. (1986) for example.

Without attempting to re-assess this distance, we note that the distance to one of the stars in our sample, RX J0255.3+1915, can be estimated reliably. In that case, the companion is much fainter than the primary (3.4 mag at $K \mathrm{~s}$ ) and its contribution to the spectral type and the unresolved aperture photometry can be neglected. The spectral type of the unresolved system is F9V, as derived from optical spectroscopy by Hearty et al. (2000a). We attribute it to the primary. Furthermore, no infrared excess was observed by Jayawardhana et al. (2001) for this source. Therefore, assuming no reddening and no near-infrared excess, we estimate the distance to RX J0255.3+1915 from the expected absolute magnitude of an F9V star. With an apparent magnitude 
$K$ s $=9.02$ (Jayawardhana et al. 2001) and an absolute magnitude $M_{K \mathrm{~s}}=2.8$ based on the stellar models of Siess et al. (2000) and the conversion table of Kenyon \& Hartmann (1995), we find a distance modulus of 6.22 , i.e., a distance of $\sim 175 \mathrm{pc}$. This distance is likely to be a lower limit because we used the absolute magnitude of a F9V dwarf (RX J0255.3+1915 is likely younger and brighter) and no extinction. Should this object be younger, it would likely be more luminous. This would increase the distance modulus and place the object further away.

\section{Disk models for LkH $\alpha 263 \mathrm{C}$}

Models of the scattered light are useful to extract the structural parameters of disks around T Tauri stars. See, e.g., Ménard $\&$ Bertout (1999) and references therein. In this section we present synthetic intensity images of dust disks in $H$-band and we compare them to the observed intensity images of $\mathrm{LkH} \alpha 263 \mathrm{C}$ obtained at CFHT in an attempt to estimate a few of its structural parameters. We also compare the same models to the Gemini $H$-band image presented by Jayawardhana et al. (2002).

The synthetic images were produced with a code that treats Mie scattering on dust particles located in a disk whose shape is described by power-laws. The density distribution is defined by the total mass as well as five geometric parameters: inner and outer radii, surface density distribution $\left(\Sigma(r) \propto r^{p}\right)$, and scale height law $\left(H(r)=H_{0}\left(r / r_{0}\right)^{\beta}\right)$. All models presented below have fixed values of $\beta=1.125, p=-1.0$, and $H_{0}=4 \mathrm{AU}$ (at a reference radius of $50 \mathrm{AU}$ ). These values are typical of other disks (e.g., Stapelfeldt et al. 1998; Stapelfeldt et al. 2002). Other models with different parameters were run but are not presented. Due to the limited angular resolution and low signalto-noise ratio in our images, model intensity maps produced with different parameters in a (small) range around these values would be also acceptable: $1.0 \leq \beta \leq 1.25 ;-0.8 \leq p \leq-1.25$; $3 \leq H_{0} \leq 8 \mathrm{AU}$ at $50 \mathrm{AU}$. Our fit to a single wavelength data set do not allow us to constrain more precisely the range of these geometrical parameters.

Dust is responsible for the scattered light. Our models include a dust size distribution where grain radii are randomly picked from a continuous size distribution. The range of radii, $a$, extend from $a_{\min }=0.03 \mu \mathrm{m}$ to $a_{\max }=0.9 \mu \mathrm{m}$ (or $0.5 \mu \mathrm{m}$ depending on the models) with a size distribution $N(a) \propto a^{-3.7}$, as derived by Mathis \& Whiffen (1989) for porous interstellar grains. The so-called "A model" from Mathis \& Whiffen (1989) was assumed to obtain the grains optical properties (i.e., refraction indices), which we set independent of grain size. The random selection of scattering particle size is weighted by the product of the number density and the scattering cross-section of all grains in the disk. Therefore, the largest grains are the most frequent scatterers although they are the less numerous.

With the parameters adopted here, the median radius of the scattering particles at a wavelength of $1.65 \mu \mathrm{m}$ ( $H$-band) is about $0.62 \mu \mathrm{m}$ when $a_{\max }=0.9 \mu \mathrm{m}$. The asymmetry parameter for this "median grain" is $g=0.73$, i.e., heavily forwardthrowing. We find that these grains are too forward-throwing to fit the image. They produce images that are peaked too much
Table 3. Parameters of the disk models.

\begin{tabular}{llllllll}
\hline \hline Panel & $\begin{array}{l}M_{\text {dust }} \\
10^{-6} M_{\odot}\end{array}$ & $\begin{array}{l}i \\
\text { deg }\end{array}$ & $\beta$ & $\mathrm{p}$ & $\begin{array}{l}H_{0} \\
\mathrm{AU}\end{array}$ & $\begin{array}{l}a_{\min } \\
\mu \mathrm{m}\end{array}$ & $\begin{array}{l}a_{\max } \\
\mu \mathrm{m}\end{array}$ \\
\hline $\mathrm{a}$ & 0.8 & 89.0 & $9 / 8$ & -1 & 4 & 0.03 & 0.9 \\
$\mathrm{~b}$ & 4.0 & 89.0 & $9 / 8$ & -1 & 4 & 0.03 & 0.9 \\
$\mathrm{c}$ & 2.4 & 88.5 & $9 / 8$ & -1 & 4 & 0.03 & 0.9 \\
$\mathrm{~d}$ & 2.4 & 89.5 & $9 / 8$ & -1 & 4 & 0.03 & 0.9 \\
$\mathrm{e}$ & 2.4 & 89.0 & $9 / 8$ & -1 & 4 & 0.03 & 0.9 \\
$\mathrm{f}$ & 2.4 & 89.0 & $9 / 8$ & -1 & 4 & 0.03 & 0.5 \\
\hline
\end{tabular}

Note: In all models $R_{\text {out }}=165 \mathrm{AU}$, valid if the distance to MBM 12 is 275 pc. For different distances, $R_{\text {out }}$ and $M_{\text {dust }}$ must to be modified.

at the center and are not bright enough at the edge of the disk. Consequently, we ran models with smaller grains, with the maximum radius cut-off in the distribution decreased from $a_{\max }=0.9 \mu \mathrm{m}$ to $a_{\max }=0.5 \mu \mathrm{m}$. In that case the "median radius" of the scattering particles is $0.37 \mu \mathrm{m}$, with $g=0.36$. These models provide a better fit to the data (see Figs. 5 and 6).

In the rest of this section, we have assumed a distance of $275 \mathrm{pc}$ to MBM 12. Should the distance to the association be smaller, the radius of the disk would be smaller. Our synthetic models can be scaled by their optical depth to yield the same intensity distribution. A small disk would have to be less massive to yield the same intensity map. Neglecting the effect of the inner radius, the extinction optical thickness in the equatorial plane of the disk scales like

$\tau_{\text {ext }} \propto \frac{M_{\text {dust }}}{H_{0} R_{\text {out }}^{1+\beta}}$.

Accordingly, should the distance to MBM 12 be 65 pc instead of $275 \mathrm{pc}$ and since we kept $\beta=9 / 8$ and $H_{0}$ fixed, the dust mass in the disk would need to be decreased by a factor $\sim 20$ to account for the smaller disk radius.

The outer radius we measured in our $H$-band images was $0.45^{\prime \prime}$ (i.e., $124 \mathrm{AU}$ at $275 \mathrm{pc}$ ), as presented in Table 2. In all models, we considered a slightly larger outer radius $R_{\text {out }}$ set at $0.6^{\prime \prime}$ (165 AU) but which is consistent with an apparent radius of $0.45^{\prime \prime}$ once noise is added. The inner radius of the disk is arbitrarily set to $0.1 \mathrm{AU}$. With these parameters set, the dust mass, the inclination and the grains properties have the largest influence on the aspect of the reflection nebulosity. Typical results are presented in Fig. 5.

With a disk radius of $165 \mathrm{AU}$, models spanning a range of dust mass between $10^{-7}$ and $10^{-4} M_{\odot}$ were calculated. Clearly the lighter model with $M_{\text {dust }}=0.8 \times 10^{-6} M_{\odot}$ does not have enough dust to produce enough extinction in the equatorial plane, hence to produce a dark lane that is wide enough with respect to the observations. See Fig. 5, panel a. We consider it a lower limit to the disk dust mass. Similarly, a model with $M_{\text {dust }}=4 \times 10^{-6} M_{\odot}$ produces a dark lane that is too wide and too deep. See Fig. 5, panel b.

The models presented in the middle and bottom rows of Fig. 5 were calculated with an intermediate mass, i.e., $M_{\text {dust }}=$ $2.4 \times 10^{-6} M_{\odot}$, in order to study the impact of the other two parameters, the inclination and the grain properties. 

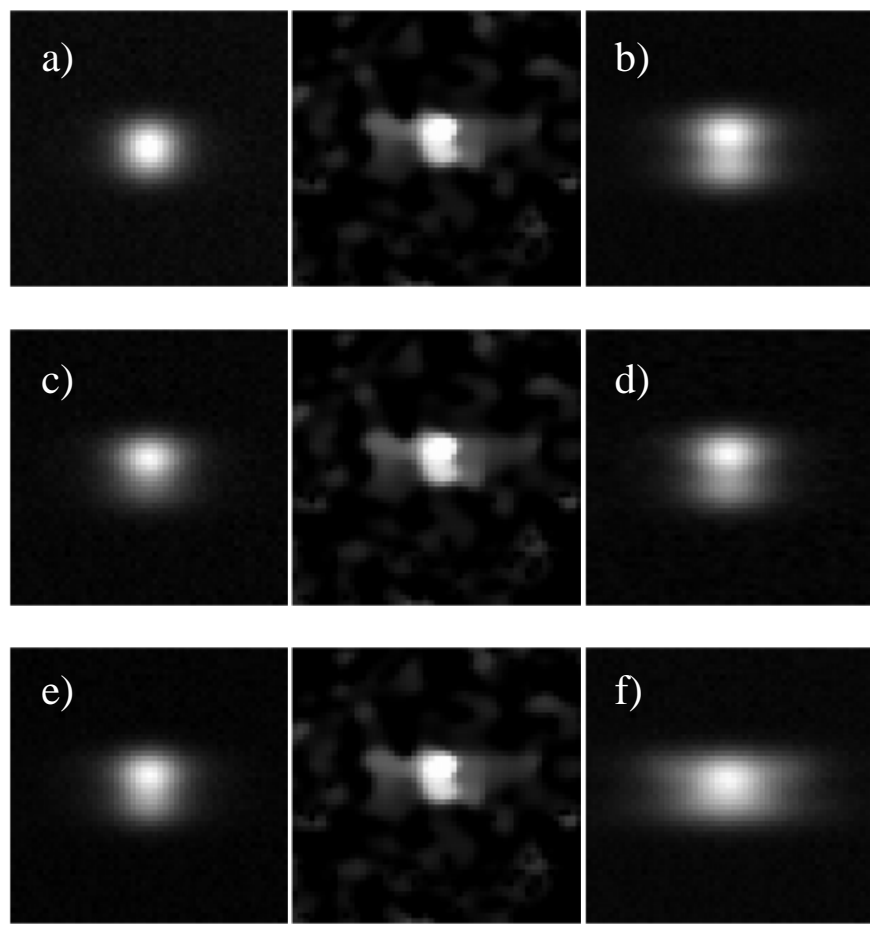

Fig. 5. Image of LkH $\alpha 263 \mathrm{C}$ obtained with PUEO at 1.65 microns and deconvolved with MISTRAL compared with scattered light models. Each model has been convolved to match the resolution of the observation. The correct read-out noise has been added to the models. Each image is presented in linear stretch. Panel a) Model with a dust mass of $8 \times$ $10^{-7} M_{\odot}$ of dust seen at $i=89^{\circ}$. Panel b) Model with a dust mass of $4 \times 10^{-6} M_{\odot}$ of dust seen at $i=89^{\circ}$. Panel c) Model with a dust mass of $2.4 \times 10^{-6} M_{\odot}$ of dust seen at $i=88.5^{\circ}$. Panel d) Model with a dust mass of $2.4 \times 10^{-6} M_{\odot}$ of dust seen at $i=89.5^{\circ}$. Panel e) Model with a dust mass of $2.4 \times 10^{-6} M_{\odot}$ of dust seen at $i=89^{\circ}$ with the nominal grain size distribution, where $a_{\max }=0.9 \mu \mathrm{m}$ (see text). Panel f) Model with a dust mass of $2.4 \times 10^{-6} M_{\odot}$ of dust seen at $i=89^{\circ}$ with the truncated grain size distribution, where $a_{\max }=0.5 \mu \mathrm{m}$ (see text). In each row, the middle panel is the observed and deconvolved intensity image obtained at CFHT. The parameters of the model presented in each panel are summarized in Table 3 .

The brightness ratio between the two nebulae on both sides of the dark lane allows to estimate the inclination of the system. Should $\mathrm{LkH} \alpha 263 \mathrm{C}$ be axisymmetric and seen dead edge-on, i.e., $i=90.0^{\circ}$, both nebulae would have the same brightness and the ratio would be unity. Estimations from the $H$-band PUEO image yields a ratio of the order of 1.3, similar to the ratio of $\sim 1.2$ found by Jayawardhana et al. (2002) at the same wavelength.

In the middle row of Fig. 5 we compare the data with models seen at an inclination of $88.5^{\circ}$ and $89.5^{\circ}$, respectively (see Fig. 5, panels c and d). Because we model disks that are only lightly flared and geometrically thin, i.e., $\beta$ and $H_{0}$ are small, the central star rapidly becomes directly visible as the inclination decreases and the contrast between the two model nebulae do not match the observations anymore. We derive an inclination of $i=89.0^{\circ}+0.5$ for a model that has $2.4 \times 10^{-6} M_{\odot}$ of dust. This model yields the proper brightness ratio and the correct separation (i.e., dark lane thickness) between the two maxima.
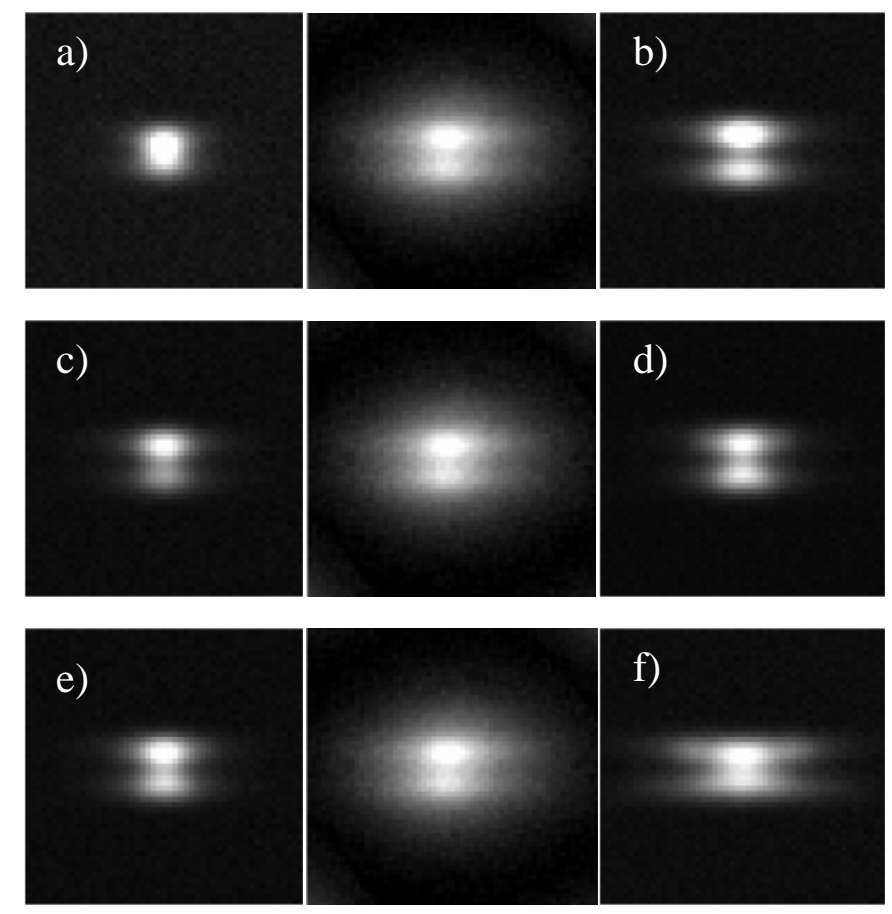

Fig. 6. Image of $\mathrm{LkH} \alpha 263 \mathrm{C}$ obtained with Gemini/Hokupa'a at 1.65 microns compared with scattered light models. The image is from Jayawardhana et al. (2002). All images are presented in linear stretch. The middle panel of each row is the data. All models have been convolved to match the resolution of the data. Panels a)-f) present the same models as in Fig. 5.

In the bottom row of Fig. 5 we probe the effect of the particle size on the brightness profile of the reflection nebulosities. Again, the models are calculated for a disk that has $M_{\text {dust }}=2.4 \times 10^{-6} M_{\odot}$. The inclination is set at $i=89.0^{\circ}$. Figure 5, panel e, shows the result when the grain distribution has a maximum cut-off radius of $0.9 \mu \mathrm{m}$. Figure 5 , panel $\mathrm{f}$, is for a distribution with a maximum cut-off radius of $0.5 \mu \mathrm{m}$. The effect is important. Given our grain size distribution, the maximum cut-off seems to be intermediate between 0.5 and $0.9 \mu \mathrm{m}$. Models with $a_{\max }=0.9 \mu \mathrm{m}$ produce images that are peaked too much at the center and do not extend far enough in the disk.

Bigger grains may be present, but they are not contributing to the scattered light significantly. Given our results, a single continuous distribution in size, extending well above micronsized particles, seems improbable because the apparent size of the disk would be too small. On the other hand, a cut-off much smaller than $0.5 \mu \mathrm{m}$ also seems ruled out because the disk surface brightness profile becomes too shallow compared to the observations and the dark lane too pronounced at the periphery, away from the center.

These affirmations do not rule out the presence of much larger grains that may have settled to the disk mid-plane. They do not rule out either well-mixed distributions where the size distribution of small grains is different than the one for the larger grains (i.e., distinct distributions).

For comparison we present the Gemini $H$-band image compared to the same models in Fig. 6. The models match these data also reasonably well. 


\section{Conclusions}

We reported the results of CFHT AO observations of the young MBM 12 association. Six binary systems were resolved, LkH $\alpha$ 264, E 0255+2018, RX J0255.4+2005, S18, MBM 1210 and RX J0255.3+1915, and one confirmed, HD 17332. A possible quadruple system composed of a close binary $\mathrm{LkH} \alpha 263 \mathrm{AB}$, a protoplanetary disk seen edge-on $\mathrm{LkH} \alpha 263 \mathrm{C}$ and a more distant star $\mathrm{LkH} \alpha 262$ was also detected. These results suggest a possible binary excess in the MBM 12 association. This may call for a revision of the spectral characterization and the IR color excess in cases where the binarity was unknown and when the contrast between companions is not large enough for one star to dominate the signal in the spectrum. New spectroscopic observations with high angular resolution are needed to obtain the spectral type. We estimate the distance to the MBM 12 candidate member RX J+0255.3+1915 at $d>175 \mathrm{pc}$.

We presented a scattered-light model of the young disk $\mathrm{LkH} \alpha 263 \mathrm{C}$ in order to extract the disk structural parameters. Our scattered light model is described by power-laws for the density distribution, the scale height and the grains size distribution. By comparing synthetic images to obervations obtained at CFHT, we derived for the disk an outer radius of $165 \mathrm{AU}$, a mass of $2.4 \times 10^{-6} M_{\odot}$, an inclination of $i=89.0_{-1.0}^{+0.5}$, and a grain distribution with a maximum cut-off radius between 0.5 and $0.9 \mu \mathrm{m}$, assuming a distance of $275 \mathrm{pc}$. If the distance is smaller, e.g. $65 \mathrm{pc}$, the outer radius is $\sim 40 \mathrm{AU}$ and $M_{\text {dust }}=1.2 \times 10^{-7} M_{\odot}$.

Acknowledgements. It is a pleasure to thank Kevin Luhman and Ray Jayawardhana for kindly making their $J H K$ data of the edge-on disk available to us before publication. All the models presented in this paper were calculated on the computers of the Service Commun de Calcul Intensif (SCCI) of the Grenoble Observatory, France. This research has made use of the SIMBAD database, operated at CDS, Strasbourg, France. We would like to thank the CNES for the postdoctoral financing of J.-C. Augereau. We also acknowledge partial financial support from the Programme National de Physique Stellaire (PNPS), in France.

\section{References}

Baraffe, I., Chabrier, G., Allard, F., \& Hauschildt, P. H. 1998, A\&A, 337,403

Conan, J.-M., Fusco, T., Mugnier, L., et al. 2000, in Adaptive Optical Systems Technology, ed. P. L. Wizinowich, Proc. SPIE, 4007, 913 Duchêne, G. 1999, A\&A, 341, 547

Duchêne, G., Bouvier, J., \& Simon, T. 1999, A\&A, 343, 831

Duquennoy, A., \& Mayor, M. 1991, A\&A, 248, 485

Fischer, D., \& Marcy, G. 1992, ApJ, 396, 178
Hartmann, L. W., \& Kenyon, S. J. 1990, ApJ, 349, 190

Hearty, T., Neuhäuser, R., Stelzer, B., et al. 2000a, A\&A, 353, 1044

Hearty, T., Fernández, M., Alcalá, J. M., Covino, E., \& Neuhäuser, R. 2000b, A\&A, 357, 681

Hobbs, L. M., Blitz, L., \& Magnani, L. 1986, ApJ, 306, 109

Hobbs, L. M., Penprase, B. E., Welty, D. E., Blitz, L., \& Magnani, L. 1988, ApJ, 327, 356

Jayawardhana, R., Luhman, K. L., D’Alessio, P., \& Stauffer, J. R. 2002, ApJ, 571, 51

Jayawardhana, R., Wolk, S. J., Barrado y Navascuès, D., Telesco, C. M., \& Hearty, T. J. 2001, ApJ, 550, L197

Kastner, J. H., Huenemoerder, D. P., Schulz, N., et al. 1997, BAAS, 191.9206

Kenyon, S. J., \& Hartmann, L. 1995, ApJS, 101, 117

Luhman, K. L. 2001, ApJ, 560, L287

Lynds, B. T. 1962, ApJS, 7, 1

Magnani, L., Blitz, L., \& Mundy, L. 1985, ApJ, 295, 402

Magnani, L., Caillaut, J.-P., \& Armus, L. 1990, ApJ, 357, 602

Mamajek, E. E., Lawson, W. A., \& Feigelson, E. D. 1999, ApJ, 516, L77

Mathis, J. S., \& Whiffen, G. 1989, ApJ, 341, 808

Mathis, J. S. 1977, ApJ, 217, 425

Ménard, F., \& Bertout, C. 1999, in The Origins of Stars and Planetary Systems, NATO Sci. Ser., Ser. C, vol. 540, ed. C. J. Lada, \& N. D. Kylafis (Kluwer: Dordrecht), 341

Ménard, F., Dougados, C., Magnier, E., et al. 2002, ApJ, submitted

Monin, J.-L., \& Bouvier, J. 2000, A\&A, 365, L75

Mouillet, D., Lagrange, A. M., Augereau, J. C., \& Ménard, F. 2001, A\&A, 372, L61

Pound, M. W., Bania, T. M., \& Wilson, R. W. 1990, ApJ, 351, 165

Siess, L., Dufour, E., \& Forestini, M. 2000, A\&A, 358, 593

Skrutskie, M. F., Dutkevitch, D., Strom, S. E., Strom, K. M., \& Shure, M. A. 1990, AJ, 99, 1187

Snowden, S. L., McCammon, D., \& Verter, F. 1993, ApJ, 409, L21

Stapelfeldt, K. R., Krist, J. E., Ménard, F., et al. 1998, ApJ, 502, L65

Stapelfeldt, K. R., Ménard, F., Watson, A. M., et al. 2002, ApJ, submitted

Torres, C. A. O., da Silva, L., Quast, G. R., de la Reza, R., \& Jilinski, E. $2000, A J, 120,1410$

Van den Ancker, M. E., Pérez, M. R., \& de Winter, D. 2000, A\&A, 363, L25

Véran, J. P. 1997, Ph.D. Thesis, Univ. Paris VII

Véran, J. P., \& Rigaut, F. J. 1998, SPIE, 3353, 426

Zimmermann, T., \& Ungerechts, H. 1990, A\&A, 238, 337

Zuckerman, B., \& Webb, R. A. 2000, ApJ, 535, 959

Zuckerman, B., Song, I., Bessell, M. S., \& Webb, R. A. 2001a, ApJ, 559,388

Zuckerman, B., Song, I. Bessell, M. S., \& Webb, R. A. 2001b, ApJ, 562, L87 